\title{
Cirugía de la comunicación interventricular post infarto: análisis de los últimos 22 años
}

\author{
Cecilia Muñoz, Sergio Morán, Andrés Jadue, Felipe León, Pedro Becker, Manuel Irarráza- \\ val, Ricardo Zalaquett.
}

División de Enfermedades Cardiovasculares. Facultad de Medicina. Pontificia Universidad

Católica de Chile. Santiago - Chile.

Introducción: El desarrollo de una comunicación interventricular en la evolución de un infarto miocárdico es una complicación muy grave, aunque infrecuente. Objetivo: Comunicar nuestros resultados con el tratamiento quirúrgico de pacientes con comunicación interventricular post infarto (CIVPI) intervenidos en los últimos 22 años.

Método: Analizamos en forma retrospectiva los antecedentes de los pacientes intervenidos entre Enero de 1991 y Diciembre de 2012. Revisamos fichas clínicas, protocolos operatorios y certificamos la mortalidad con el Registro Civil e Identificación de Chile.

Resultados: Operamos 43 pacientes, edad promedio de 66,6 $6 \pm 10,2$ años. El 58\% eran hombres, de menor edad que las mujeres $(63,1 \pm 10,8$ vs $71,5 \pm 6,9$ años, $\mathrm{p}=0,006$ ). El tiempo promedio entre el diagnóstico de infarto y de CIVPI fue $10 \pm 15$ días. El $74 \%$ fueron intervenidos de urgencia. La CIVPI fue anterior en 58\%. Se realizó revascularización miocárdica concomitante en el $58 \%$. Trece pacientes fallecieron (30\%) en el posoperatorio. Factores de riesgo de mortalidad operatoria fueron: cirugía de urgencia $(\mathrm{p}=0,019])$ y uso de balón intra aórtico $(\mathrm{p}=0,006)$. La cirugía realizada después de las 24 horas del diagnóstico tuvo una mortalidad significativamente menor $(7,7 \%, \mathrm{p}=0,033)$. El seguimiento promedio fue de 8,36 $\pm 5,3$ años. La supervivencia alejada, excluida la mortalidad operatoria, a 5 y 10 años, fue $93 \%$ y $71 \%$, respectivamente.

Conclusión: La CIVPI tiene elevada mortalidad operatoria, especialmente en pacientes intervenidos de urgencia y en los que requirieron balón de contra pulsación, pero la supervivencia alejada de los sobrevivientes es muy satisfactoria.

Correspondencia:

Ricardo Zalaquett S.

División de Enfermedades Cardiovasculares

Pontificia Universidad Católica de Chile

rzalaque@med.puc.cl 


\section{Surgery for post infarction ventricular septal defect: a 22-year experience}

Background: The development of a ventricular septal defect (VSD) after myocardial infarction is a rare but very serious complication for which the treatment of choice is surgical repair.

Aim: To report our results with patients operated on for post -infarction VSD in the last 22 years.

Methods: This is a retrospective review of all patients operated for post infarction VSD between january 1991 and december 2012. We reviewed all clinical charts and operative notes. Long-term mortality was certified by the "Registro Civil e Identificación de Chile".

Results: Fourty three patients with a mean age of $66.6 \pm 10.2$ years underwent surgical repair. Fifty eight percent were males. The average time between myocardial infarction and the diagnosis of vsd was 10 \pm 15.2 days. Seventy four percent of patients were operated on as an emergency. In 58\% of cases the VSD was located in the anterior septum. Myocardial revascularization was performed in $58 \%$. Thirteen patients died for an operative mortality of $30 \%$. Risk factors for operative mortality were emergency surgery $(p=0,04)$ and the use of intra-aortic balloon pump $(p=0,004)$. Non emergency surgery had a much lower mortality rate $(7,7 \%, \mathrm{p}=0,033)$. Survival excluding operative mortality at 5 and 10 years was $90 \%$ and $71 \%$, respectively.

Conclusions: Operative mortality for repair of post infarction VSD remains high, mainly in patients undergoing an emergency operation. Surgical survivors have a very good life expectancy.

Key words: Myocardial infarction, ventricular septal rupture, cardiac surgery. 


\section{Introducción}

El desarrollo de una comunicación interventricular durante la evolución de un infarto es una complicación muy grave, aunque infrecuente ${ }^{1-2}$. Su incidencia se estima entre $0,2 \%$ a $3 \%$ de todos los pacientes con infarto miocárdi$\mathrm{co}^{1-3}$. La mortalidad con tratamiento médico exclusivo es sobre $90 \%$, por lo cual el tratamiento de elección es su cierre quirúrgico ${ }^{4-5}$. Sin embargo, la intervención también tiene una mortalidad operatoria de consideración, variando entre $19 \%$ a $60 \%{ }^{1-6}$.

La CIVPI se ubica en el septum anterior en aproximadamente el $60 \%$ de los casos, debido a oclusión de la arteria descendente anterior e infarto ántero septal. En el $40 \%$ restante, se ubica en el septum posterior por compromiso de la arteria descendente posterior o de ramas circunflejas. Los parámetros hemodinámicos, como la hipoperfusión sistémica, la magnitud del cortocircuito de izquierda a derecha y el tamaño del infarto, son los factores determinantes del pronóstico de esta grave complicación, que es responsable de aproximadamente el $5 \%$ de las muertes precoces por infarto miocárdico ${ }^{1-6}$.

La CIVPI tiene un cuadro clínico de presentación similar caracterizado por deterioro hemodinámico y aparición de un soplo holosistólico. El diagnóstico diferencial principal es con la insuficiencia mitral isquémica, ya sea por rotura de músculo papilar, o funcional, por tracción de los velos mitrales, secundaria a la disyunción y dilatación del ventrículo izquierdo ${ }^{2-5-7-8}$.

El objetivo del presente trabajo fue determinar la incidencia quirúrgica las características clínicas y anatomo-patológicas, la mortalidad operatoria y la supervivencia alejada de los pacientes operados por CIVPI en nuestra institución.

\section{Método y pacientes}

Revisamos en forma retrospectiva la base de datos del Servicio de Cirugía Cardiovascular del Hospital Clínico de la Pontificia Universidad Católica de Chile entre Enero de 1991 y Diciembre de 2012. Se analizaron las características clínicas pre-operatorias y quirúrgicas, la incidencia de cortocircuito residual significativo y la mortalidad operatoria (hasta 30 días de la cirugía). La supervivencia alejada se confirmó a través del Registro Civil e Identificación de Chile.

Este análisis retrospectivo fue aprobado por el comité de ética de la institución.

Durante el período en estudio, 43 pacientes fueron operados con diagnóstico de CIVPI. Para la cirugía se utilizó circulación extracorpórea con oxigenador de membrana e hipotermia sistémica entre $28^{\circ}$ y $30^{\circ} \mathrm{C}$. La protección mio- cárdica se efectuó con solución cardioplégica cristaloide infundida a $4^{\circ} \mathrm{C}^{9}$.

Las técnicas quirúrgicas utilizadas fueron el cierre con parche, la exclusión del área infartada y de la rotura del septum con parche amplio, la amputación del ápex y el cierre con dispositivo Amplatzer $\AA$.

La eficacia del cierre de la comunicación fue evaluada en todos los casos al término de la circulación extracorpórea mediante ecocardiografía transesofágica.

Estadística: Las variables continuas son expresadas como promedio \pm desviación estándar y las variables categóricas como frecuencias. Se compararon edades con test de ANOVA. Las variables asociadas a mortalidad fueron analizadas con la prueba de $\mathrm{Chi}^{2}$ y la prueba de Fischer. La supervivencia alejada fue estimada con la prueba de Kaplan-Meier y la comparación de variables asociadas a la supervivencia con la prueba de Breslow o Log-Rank. Se utilizó el programa SPSS 15.0 y se consideró significativo un valor $\mathrm{p}<0,05$.

\section{Resultados}

Incidencia Quirúrgica: Durante el período de estudio intervenimos a 6.183 pacientes por enfermedad coronaria, de los cuales 43 lo fueron por CIVPI. Esto representa el $0,6 \%$ del total de pacientes con enfermedad coronaria operados en nuestra. La incidencia anual fue muy variable, entre ninguno a 5 pacientes al año.

Características Clínicas y Quirúrgicas: La edad promedio de los pacientes fue 66,6 \pm 10,2 años (margen 45-84 años). Veinticinco pacientes eran hombres (58\%) de menor edad que las mujeres $(63,1 \pm 10,8, \mathrm{vs} 71,5 \pm 6,9, \mathrm{p}=$ 0,006). La mitad de los pacientes tenían 70 o más años (21 casos). El $42 \%$ de los pacientes eran hipertensos, $40 \%$ fumadores, $23 \%$ diabéticos y $19 \%$ dislipidémicos. El tiempo de evolución del infarto al diagnóstico de la CIVPI fue en promedio de $10 \pm 15,2$ días, con una mediana de 5 días y un margen de 1 a 60 días. Seis pacientes fueron tratados con trombolisis en las primeras horas de evolución del infarto. Treinta y dos pacientes (74\%) fueron operados de urgencia. En 26 pacientes $(61 \%)$ se utilizó un balón de contrapulsación intra aórtico (BCIA) (Tabla 1).

En 25 pacientes (58\%) la lesión se ubicó en el septum anterior y en los otros 18 (42\%) en el posterior. El abordaje quirúrgico a la rotura septal fue a través de una ventriculotomía izquierda, en la zona del infarto, en 39 pacientes $(91 \%)$ y a través de una auriculotomía derecha en 2 $(4,7 \%)$. Principalmente, se utilizó la técnica de cierre con parche $(44 \%)$ y, exclusión de la rotura septal y de la zona infartada con un parche amplio (40\%) (Tabla 2). 


\begin{tabular}{|c|c|c|}
\hline \multicolumn{3}{|c|}{$\begin{array}{l}\text { Tabla 1: Características clínicas de } 43 \text { pacientes } \\
\text { operados por comunicación interventricular post infarto } \\
\text { del miocardio. }\end{array}$} \\
\hline Hombres & $25(58 \%)$ & \\
\hline Edad años, promedio \pm ds (margen) & $66,6 \pm 10,2(45-84)$ & \\
\hline Hombres & $63,1 \pm 10,8$ & $p=0,006$ \\
\hline Mujeres & $71,5 \pm 6,9$ & \\
\hline Mayor 70 años & $21(49 \%)$ & \\
\hline Hipertensión Arterial & $18(42 \%)$ & \\
\hline Diabetes Mellitus & $10(23 \%)$ & \\
\hline Tabaquismo & $17(40 \%)$ & \\
\hline Dislipidemia & $8(19 \%)$ & \\
\hline \multicolumn{3}{|l|}{ Tiempo entre diagnóstico de IAM y cirugía } \\
\hline Primeras 24 horas & $12(28 \%)$ & \\
\hline Después 24 horas & $31(72 \%)$ & \\
\hline \multicolumn{3}{|l|}{ Tiempo entre diagnóstico de CIV y cirugía } \\
\hline Primeras 24 horas & $29(67 \%)$ & \\
\hline Después de 24 horas & $14(33 \%)$ & \\
\hline Urgencia & $32(74 \%)$ & \\
\hline Uso BCPA & $26(61 \%)$ & \\
\hline Shock cardiogénico & $20(47 \%)$ & \\
\hline Coronariografía pre-operatoria & $36(84 \%)$ & \\
\hline \multicolumn{3}{|l|}{ Ubicación de la rotura septal } \\
\hline Anterior & $25(58 \%)$ & \\
\hline Posterior & $18(42 \%)$ & \\
\hline $\begin{array}{l}\text { Otras roturas asociadas } \\
\text { (músculo papilar, pared libre) }\end{array}$ & $4(9,3 \%)$ & \\
\hline
\end{tabular}

En 25 pacientes (58\%) se efectuó una revascularización miocárdica con un promedio de 1,8 \pm 1 puentes aortocoronarios (margen 1 a 4). En 21 pacientes se efectuó la revascularización de la zona infartada. Dos pacientes presentaron rotura de pared libre y uno de músculo papilar, otro paciente presentó una rotura de pared libre y de músculo papilar, además de la CIVPI. La rotura de pared libre fue reparada con cierre directo sobre bandas de teflón y reforzamiento de toda la zona infartada con parche de pericardio bovino. En los 2 casos de rotura de músculo papilar se efectuó un reemplazo valvular mitral con prótesis mecánica.

Tabla 2: Técnica quirúrgica utilizada en 43 pacientes.

\begin{tabular}{|lrr|}
\hline Técnica Quirúrgica & No $^{\circ}$ & $\%$ \\
\hline Cierre directo con parche & 19 & 44 \\
\hline Cierre y exclusión del infarto con parche & 17 & 40 \\
\hline Cierre Primario & 4 & 9 \\
\hline Amputación del Ápex & 2 & 4,7 \\
\hline Cierre trans ventricular con Amplatzer $®$ & 1 & 2,3 \\
\hline
\end{tabular}

Tabla 3: Factores asociados a mortalidad operatoria.

\begin{tabular}{|c|c|c|c|}
\hline & Supervivientes & Fallecidos & $p$ \\
\hline$n$ & 30 & 3 & \\
\hline Sexo & & & 0,752 \\
\hline Hombres & $18(72 \%)$ & $7(28 \%)$ & \\
\hline Mujeres & $12(66,7 \%)$ & $6(33,3 \%)$ & \\
\hline Edad (Años) & $65,4 \pm 9,2$ & $69,5 \pm 12$ & 0,230 \\
\hline Localización CIV & & & 0,747 \\
\hline Anterior & $18(72 \%)$ & $7(28 \%)$ & \\
\hline Posterior & $12(66,6 \%)$ & $6(33,3 \%)$ & \\
\hline Evolución Infarto (días) & $12,1 \pm 17,4$ & $4,9 \pm 4,6$ & 0,154 \\
\hline Cirugía de Urgencia & $19(59,4 \%)$ & $13(40,6 \%)$ & 0,019 \\
\hline BCPA & $14(53,8 \%)$ & $12(46,2 \%)$ & 0,006 \\
\hline Tipo Reparación & & & $\mathrm{p}=0,230$ \\
\hline Cierre con Parche & $14(32,6 \%)$ & $5(11,6 \%)$ & \\
\hline Parche y Exclusión & $11(25,6 \%)$ & $6(25,6 \%)$ & \\
\hline Cierre Primario & $3(7 \%)$ & $1(2,3 \%)$ & \\
\hline Amputación Ápex & $1(2,3 \%)$ & $1(2,3 \%)$ & \\
\hline Cierre con Amplatzer $®$ & $1(2,3 \%)$ & 0 & \\
\hline $\begin{array}{l}\text { Cirugía en primeras } 24 \text { hrs } \\
\text { de dg CIVPI }\end{array}$ & $17(58,6 \%)$ & $12(41,4 \%)$ & $p=0,033$ \\
\hline $\begin{array}{l}\text { Revascularización } \\
\text { asociada }\end{array}$ & $15(60 \%)$ & $10(40 \%)$ & $\mathrm{p}=0,178$ \\
\hline
\end{tabular}

BCIA: Balón de Contrapulsación Intra Aórtico;

CIVPI: Comunicación Interventricular post infarto.

Mortalidad Operatoria: Trece pacientes fallecieron en el período peri-operatorio (30\%). Los factores asociados a mortalidad operatoria fueron la cirugía de urgencia (11 de $27, p=0,04$, OR 6,9, IC 95\% 0,77- 6,1) y el uso de balón de contra pulsación intra aórtico (11 de 23, p = 0,004, OR 12,8, IC 95\%, 1,44 - 114,4) La cirugía realizada después de las 24 horas del diagnóstico tuvo una mortalidad significativamente menor, 7,7\% ( 1 de 13, p = 0,033, OR 0,109, IC 95\% 0,013-0,949); (Tabla 3). El sexo no influyó en la mortalidad operatoria, a pesar que la edad de las mujeres fue significativamente mayor.

CIVPI residual: Sólo dos pacientes evolucionaron con una CIV residual significativa por la que debieron ser re-intervenidos en nuestra institución (2/30, 6,7\%).

Supervivencia Alejada: El promedio de tiempo entre la cirugía y la constatación de sobrevida o fallecimiento fue de $8,4 \pm 5,3$ años con una mediana de 8 años y un margen de 0,2 a 18 años. Al término de este período 16 pacientes estaban vivos. La supervivencia a 1,5 y 10 años fue de $65,1 \% \pm 7,3 \%, 62,5 \% \pm 7,4 \%$ y $49,4 \% \pm 8,3 \%$, respecti- 
vamente (figura 1). Excluida la mortalidad operatoria, la supervivencia a 1,5 y 10 años fue de $93,3 \% \pm 4,6 \%, 89,6 \%$ $\pm 5,7 \%$ y $70,8 \% \pm 9,5 \%$, respectivamente (figura 2 ). No encontramos factores que se relacionaran estadísticamente con la supervivencia.

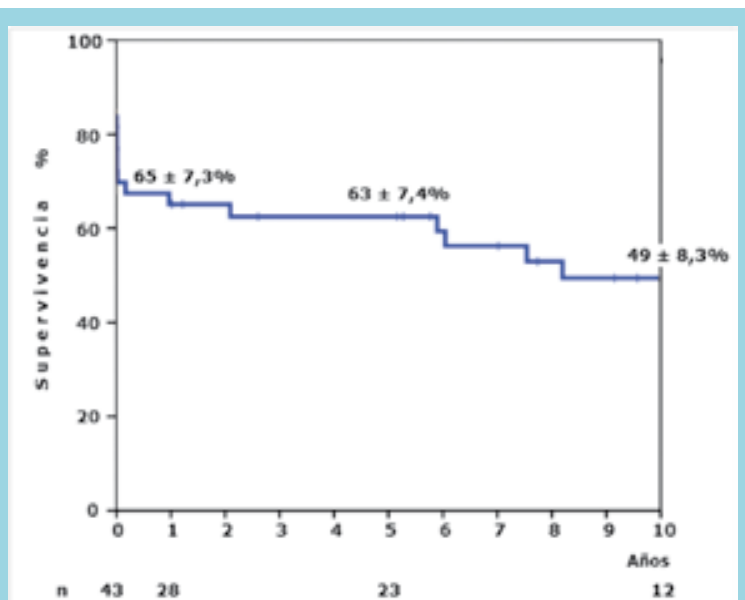

Figura 1: Supervivencia global de pacientes operados.

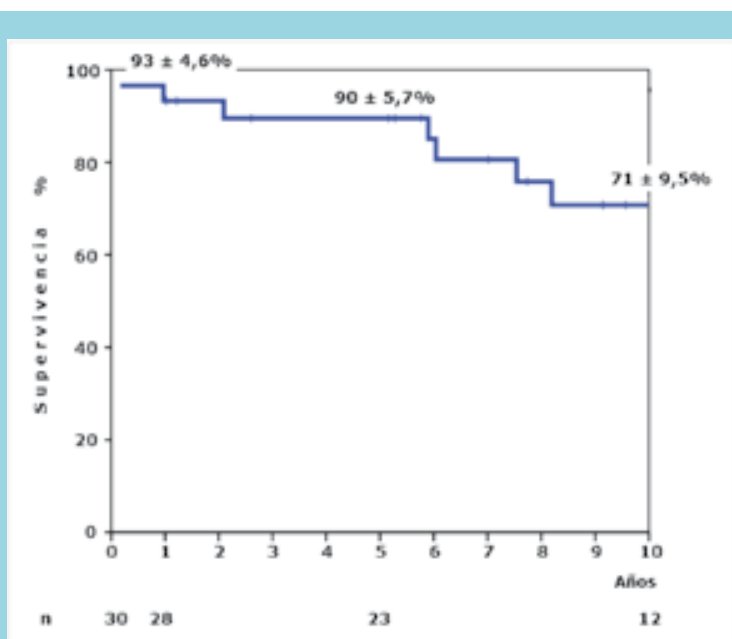

Figura 2: Supervivencia de pacientes operados excluida mortalidad operatoria.

\section{Discusión}

La primera descripción de una CIVPI fue efectuada en una autopsia a mediados del siglo XIX. Setenta años más tarde se efectúa el diagnóstico clínico de la complicación y se definen los criterios para su diagnóstico y su relación con la enfermedad coronaria ${ }^{10}$. Finalmente en 1956, Cooley comunica el primer cierre quirúrgico exitoso de esta condición ${ }^{11}$.

La extrema gravedad de esta complicación del infarto mio-
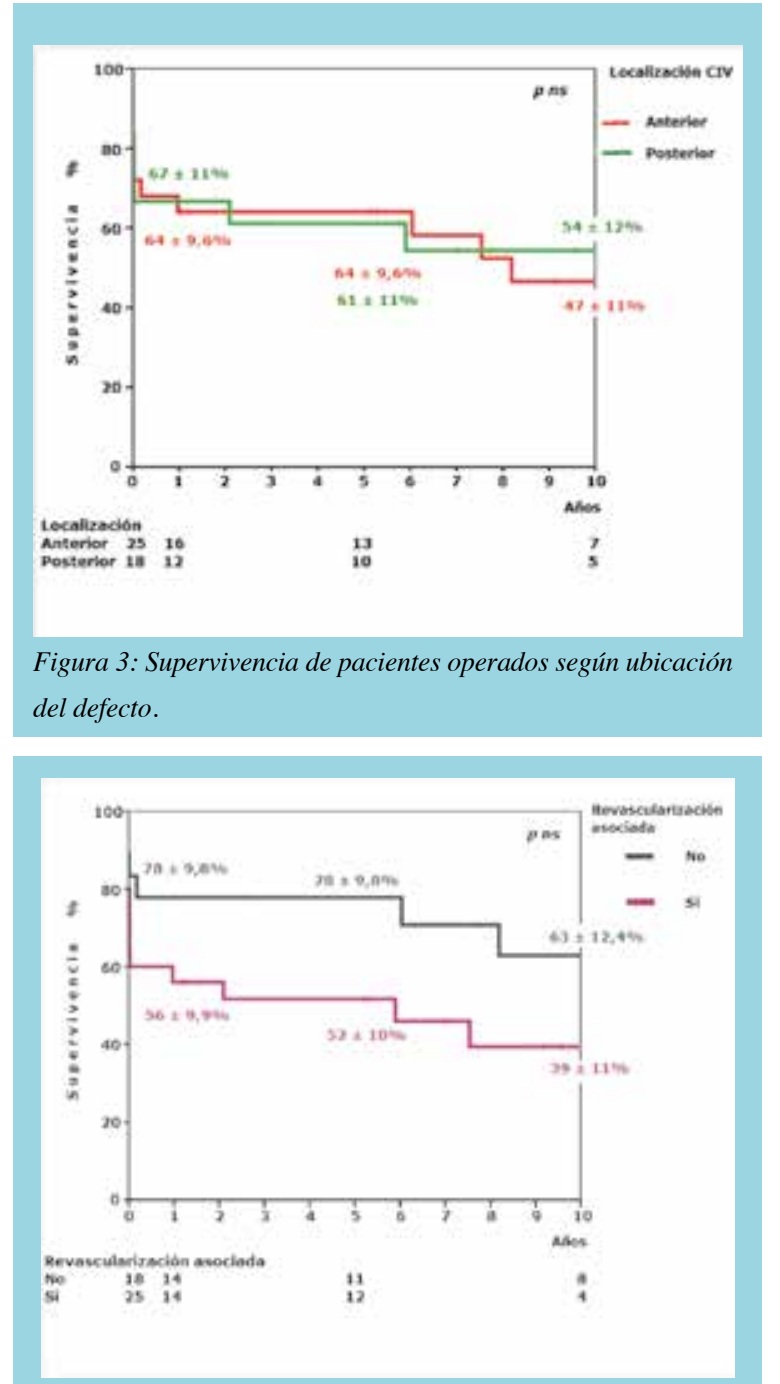

Figura 4: Supervivencia de pacientes operados según presencia de revascularización miocárdica asociada.

cárdico ha mantenido un permanente interés en ella, pero su baja incidencia ha dificultado definir bien su historia natural y los factores asociados al pronóstico quirúrgico, existiendo aún discusión en muchos de estos aspectos. Por otra parte, estas mismas características hacen que no sea posible llevar a cabo estudios comparativos y aleatorios, por lo que la información se basa en experiencias clínicas. Incidencia. Previo a la terapia trombolítica inicial del infarto, la CIVPI se presentaba en el $1 \%$ a $3 \%$ de ellos y era responsable del $5 \%$ de las muertes precoces ${ }^{2-12-14}$. Después de la introducción de la reperfusión precoz del infarto con trombolíticos, su incidencia disminuyó a 0,2\%1. En Chile, es difícil estimar la real incidencia de la CIVPI. En nuestra experiencia tiene una incidencia de $0,6 \%$ de los pacientes operados por enfermedad coronaria. 
Las características demográficas de nuestros pacientes son muy similares a una publicación reciente de la base de datos de la "Society of Thoracic Surgeons" (STS) $)^{15}$, que comprendió un total de 2.876 pacientes operados por CIVPI: el 56,5\% de los pacientes eran hombres; en nuestra serie $58 \%$. El promedio de edad fue $68 \pm 11$ años; en la nuestra $67 \pm 10$ años. No hay referencia de edad según sexo en la publicación, pero en nuestra serie la edad de ocurrencia en mujeres fue significativamente mayor que en los hombres $(63,1 \pm 10,8$ vs 71,5 $\pm 6,9, \mathrm{p}=0,006)$.

Características Clínicas y Quirúrgicas. Como en la mayoría de las series, la rotura septal se produjo frecuentemente durante la primera semana del infarto ${ }^{2,12-14}$. En algunos pacientes la complicación se presentó muy precozmente, probablemente secundaria al uso de trombolíticos. El estudio GUSTO I, mostró que si bien disminuyó la incidencia de la complicación, se favoreció la rotura precoz del septum ${ }^{1}$.

Como en otras experiencias recientes, la mayoría de nuestros pacientes fueron operados de urgencia, es decir, durante las 24 horas de efectuado el diagnóstico de CIVPI. En estos casos el apoyo con balón intra aórtico de contrapulsación por deterioro hemodinámico, es necesario y constituye una indicación aceptada ${ }^{3}$. Nosotros lo usamos en $61 \%$ de los pacientes. Igualmente, la localización anterior del defecto fue el más frecuente ${ }^{15-17}$.

La técnica quirúrgica de exclusión de la comunicación y de la zona infartada con un parche de pericardio bovino descrita por David, es la más utilizada en la actualidad ${ }^{18}$. Una alternativa emergente es el cierre de la CIVPI por vía percutánea transfemoral o por vía perventricular, con un dispositivo Amplatzer®. En nuestra serie, este procedimiento se realizó en un paciente, considerado extremadamente grave para la cirugía tradicional. Esta técnica, aunque atractiva, tiene aún limitaciones relacionadas con las características anatómicas de la CIVPI y la solidez de los tejidos. Se ha comunicado un uso más eficaz de esta técnica en casos de un defecto septal residual ${ }^{19-21 .}$

En el 58\% de nuestros pacientes se efectuó revascularización miocárdica asociada, lo que es algo menor a la reportada, que se aproxima al $70 \%{ }^{15}$. El número de puentes fue 1,8/paciente, mayor que lo publicado. Probablemente, ésto traduce la tendencia a no revascularizar la zona infartada, lo que sólo se efectuó en el $47 \%$ de los casos ${ }^{15-17}$.

Las publicaciones más recientes no comunican rotura del ventrículo izquierdo asociada a la CIVPI, que en nuestra serie se presentó en 4 pacientes. La serie del STS señala una incidencia de procedimientos mitrales simultáneos de $7,3 \%$, semejante a nuestra experiencia ${ }^{15}$.
Mortalidad Operatoria. La mortalidad operatoria en nuestra serie $30 \%$, fue menor que en la mayoría de las experiencias actuales ${ }^{15-17}$. Específicamente, en el estudio de la base de datos del STS, que incluyó un gran número de pacientes proveniente de múltiples centros, la mortalidad operatoria fue $43 \% 15$.

En varias series la urgencia ha sido el principal factor de riesgo, con una mortalidad cercana al $60 \%{ }^{15,16}$. Esto ha producido un debate en cuanto al mejor momento para indicar la cirugía. Las guías clínicas del American College of Cardiology-American Heart Association recomiendan la intervención quirúrgica inmediata de los pacientes con CIVPI $^{3}$. Sin embargo, una alternativa para los pacientes de mayor riesgo sería la conexión a un sistema de asistencia ventricular, que permitiría diferir la cirugía en espera de mejores condiciones anatómicas y fisiológicas ${ }^{15-22}$. Nuestros resultados con pacientes operados mas tardíamente fue significativamente mejor, lo que apoyaría esta proposición.

El balón intra aórtico de contrapulsación se asocia a una mayor mortalidad operatoria, lo que se explica porque su uso está indicando mayor compromiso miocárdico y mayor inestabilidad hemodinámica ${ }^{2-5-6-15}$.

La revascularización miocárdica concomitante a la reparación de la CIVPMI no influyó en el pronóstico vital en nuestra serie. Este es un factor no aclarado ya que hay series en que sí se asoció a mejor pronóstico ${ }^{23-24}$, en tanto que en otras no lo fue ${ }^{15-16}$.

En nuestra experiencia la ubicación anatómica del defecto no fue estadísticamente determinante para la mortalidad operatoria, lo que difiere de otras series en que la ubicación posterior se asoció a una mayor mortalidad ${ }^{2,16}$. Esto es esperable dado que el infarto de pared posterior se acompaña de infarto del ventrículo derecho y su cierre quirúrgico es técnicamente más difícil ${ }^{2,5}$. Una explicación de esta discrepancia puede ser el limitado número de casos de nuestra serie.

Defecto Residual. La incidencia de flujo residual a través del septum determinada ecocardiográficamente, información que no fue posible obtener en todos nuestros pacientes, se estima en general entre $25 \%$ a $30 \%{ }^{2}$. Sin embargo, la incidencia de cortocircuito hemodinámicamente significativo, que requiere de reintervención, es sustancialmente menor, solo algo mayor a la nuestra que fue $6,7 \%$ 2-16-17-25. Supervivencia Alejada. La supervivencia a 5 años en la mayoría de las series es algo menor a la nuestra, entre $40 \%$ y $60 \%$. Sin embargo, el mayor determinante de esta limitada supervivencia es la elevada mortalidad operatoria $^{1-2-6-16-17}$. La supervivencia excluída la mor- 
talidad fue buena en nuestra serie: $90 \%$ a 5 años y $71 \%$ a 10 años.

Al igual que en la mortalidad precoz, la técnica quirúrgica utilizada, la revascularización asociada, y la ubicación de la CIV, no influyeron en la supervivencia alejada (figuras 3 y 4). La cirugía de urgencia y la revascularización miocárdica se asociaron a un aumento considerable de la mortalidad operatoria. Por otro lado, la cirugía realizada después de las primeras 24 hrs., de realizado el diagnóstico se asoció a un menor riesgo vital. Si bien, siempre ha habido controversia respecto a si la revascularización miocárdica mejora el pronóstico de la CIV post-infarto ${ }^{23,24}$, nuestros resultados son similares al estudio sueco en el que la cirugía coronaria tendió a empeorar la supervivencia alejada e incluso el número de anastomosis coronarias resultó ser un predictor independiente de mortalidad aleja$\mathrm{da}^{16}$. Una explicación sería que si bien es de esperar que la revascularización miocárdica tenga un efecto protector, un mayor número de anastomosis estaría traduciendo una enfermedad coronaria más severa. Como quiera que sea, la recomendación sigue siendo efectuar una revascularización miocárdica completa, lo que no implica, por otra parte, que sea mandatorio revascularizar el vaso ocluido, responsable del infarto y de la $\mathrm{CIV}^{3}$.

Limitaciones. La principal limitación de este estudio es su carácter retrospectivo. Como ya se señaló, efectuar un estudio prospectivo, aleatorio y ciego para esta patología resulta prácticamente imposible. Una segunda limitación es que representa la experiencia de un solo centro, por lo que sus resultados no son necesariamente extrapolables a todo el país. Finalmente, este estudio carece de un control clínico y ecocardiográfico alejado de todos los supervivientes.

\section{Conclusión}

La comunicación interventricular es una complicación grave pero infrecuente del infarto miocárdico. La incidencia es mayor en hombres en quienes, a diferencia de las mujeres, ocurre a menor edad. La cirugía es la mejor alternativa terapéutica, aunque la mortalidad operatoria es todavía elevada, siendo la urgencia el principal factor de riesgo. Sin embargo, los sobrevivientes de la cirugía tienen una muy buena expectativa de vida.

\section{Agradecimientos}

A los doctores Juan Dubernet y Gustavo Maturana, por aportar a este estudio pacientes operados por ellos.

\section{Referencias:}

1. CRENSHAW BS, GRANGER CB, BIRMBAUM Y, PIEPER KS, MORRIS DC, KLEIMAN NS, et al. Risk factors, angiographic patterns, and outcomes in patients with ventricular septal defect complicating acute myocardial infarction. GUSTO-I (Global Utilization of Streptokinase and TPA for Occluded Coronary Arteries). Trial Investigators. Circulation 2000;101:27-32.

2. BIMBAUM Y, FISHBEIN MC, BLANCHE C, SIEGEL RJ. Ventricular septal ruptura after acute myocardial infarction. N Engl J Med 2002;347:1426-32.
3. RYAN TJ, ANTMAN EM, BROOKS NH, CALIFF RM, HILLIS LD, HIRATZKA LF, et al. 1999 update: ACC/AHA guidelines for the management of patients with acute myocardial infarction. A report of the American College of Cardiology/ American Heart Association Task Force on Practice Guidelines (Committee on Management of Acute Myocardial Infarction) J Am Coll Cardiol 1999;34:890-911.

4. DAVID TE, ARMSTRONG S. Surgical repair of postinfarction ventricular septal defect by infarct exclusion. Semin Thorac Cardiovasc Surg 1998;10:105-10. 
5. LABROUSSE L, CHOUKROUN E, CHAVALIER JM, MADONNA M, ROBERTIE F, MERLICO F, et al. Surgery for post infarction ventricular septal defect (VSD): risk factors for hospital death and long term results. Eur J Cardiothorac Surg 2002;21:725-31.

6. CERIN G, DI DONATO M, DIMULESCU D, MONTERICCIO V, MENICANTI L, FRIGIOLAA, et al. Surgical treatment of ventricular septal defect complicating acute myocardial infarction. Experience of a north Italian referral hospital. Cardiovasc Surg 2003;11:149-54.

7. ZALAQUETT R, CARTAJENA F, BECKER P, IRARRÁZAVAL M, MORAN S. Cirugía de la rotura de músculo papilar por infarto miocárdico. Rev Med Chile 2009;137:25-30.

8. ZALAQUETT R, FLORES E, CARTAJENA F, BACHLER P, CÓRDOVA S, BECKER P, et al. Resultados alejados de la cirugía reparadora de la insuficiencia mitral isquémica. Rev Med Chile 2011;139:1544-1552.

9. ZALAQUETT R, BECKER P, IRARRÁZAVAL M, MORÁN S, MATURANA G, NAVARRO M, et al. Cardioplegia retrógrada por canulación transauricular derecha del seno coronario. Rev Chil Cardiol 1993; 12: 68-71.

10. SAGER R. Coronary thrombosis: perforation of the infarcted interventricular septum. Arch Intern Med 1934; 53: 140.

11. COOLEY DA, BELMONTE BA, ZEIS LB, SCHNUR S. Surgical repair of ruptured interventricular septum following acute myocardial infarction. Surgery 1957; 41: 930.

12. POHJALA-SINTONEN S, MULLER JE, STONE PH, WILLICH SN, ANTMAN EM, DAVIS VG, et al. Ventricular septal and free wall ruptura complicating acute myocardial infarction: experience in the Multicenter Investigation of Limitation of Infarct Size. Am Heart J 1989;117:809-18.

13. TOPAZ O, TAYLOR AL. Interventricular septal ruptura complicating acute myocardial infarction: from pathophysiologic features to the role of invasive and noninvasive diagnostic modalities in current management. Am J Med 1992; 93: 683-8.

14. MOORE CA, NYGAARD TW, KAISER DL, COOPER AA, GIBSON RS. Postinfarction ventricular septal rupture: the importance of location of infarction and right ventricular function in determining survival. Circulation 1986;74:45-55.

15. ARNAOUTAKIS GJ, ZHAO Y, GEORGE TJ, SCIORTINO CM, MCCARTHY PM, CONTE JV. Surgical Repair of ventricular septal defect after myocardial infarction: outcomes from the Society of Thoracic Surgeons National Database. Ann Thorac Surg 2012; 94: 436-44.
16. JEPPSSON A, LIDEN H, JOHNSSON P. HARTFORD M, RADEGRAN K. Surgical repair of post infarction ventricular septal defects: a national experience. Eur J Cardiothoracic Surg 2005; 27: 216-21.

17. FUKUSHIMA S, TESAR PJ, JALALI H, CLARKE AJ, SHARMA H, CHOUDHARY J. et al. Determinant of in-hospital and long-term surgical outcomes after repair of postinfarction ventricular septal rupture. J Thorac Cardiovasc Surgery 2010; 140: 59-65.

18. DAVID TE, DALE L, SUN Z. Postinfarction ventricular septal ruptura: repair by endocardial patch with infarct exclusion. J Thorac Cardiovasc Surg 1995; 110: 1315-22.

19. HOLZER R, BALZER D, AMIN Z, RUIZ CE, FEINSTEIN J, BASS J, et al. Transcatheter closure of postinfarction ventricular septal defects using the new Amplatzer muscular VSD occluder: results of a U.S. Registry. Catheter Cardiovasc Interv 2004;61:196-201.

20. LEE MS, KOZITZA R, MUDRICK D, WILLIAMS M, LODGE AJ, HARRISON JK, et al. Intraoperative device closure of postinfarction ventricular septal defects. Ann Thorac Surg 2010; 89: e48-50.

21. MALTAIS S, IBRAHIM R, BASMADJIAN AJ, CARRIER M, BOUCHARD D, CARTIER R, et al. Postinfarction ventricular septal defects: towards a new treatment algorithm? Ann Thorac Surg 2009;87:687-92.

22. CONRADI L, TREEDE H, BRICKWEDEL J, REICHENSPURNER H. Use of initial biventricular mechanical support in a case of postinfarction ventricular septal ruptura as a bridge to surgery. Ann Thorac Surg 2009; 87: e37-9.

23. MUEHRCKE DD, DAGGETT WM JR, BUCKLEY MJ, AKINS CW, HILGENBERG AD, AUSTEN WG. Postinfarct ventricular septal defect repair: effect of coronary artery bypass grafting. Ann Thorac Surg 1992; 54: 876-82.

24. BARKER TA, RAMNARINE IR, WOO EB, GRAYSON AD, AU J, FABRI BM, et al. Repair of post-infarct ventricular septal defect with or without coronary artery bypass grafting in the northwest of England: a 5-year multi-institutional experience. Eur J Cardiothorac Surg 2003; 24: 940-6.

25. SKILLINGTON PD, DAVIES RH, LUFF AJ, WILLIAMS JD, DAWKINS KD, CONWAY N, et al. Surgical treatment for infarct-related ventricular septal defects: improved early results combined with analysis of late functional status. J Thorac Cardiovasc Surg 1990; 99: 798-808. 